\title{
Factores de riesgo de infección postcesárea Hospital Universitario de Caldas
}

\author{
Mario Arturo González Mariño*; Eduardo León Jaramillo Velásquez**; Carlos Alberto Esparza Duque***
}

\begin{abstract}
RESUMEN: En el Hospital Universitario de Caldas, Manizales, Colombia, se adelantó entre el 1 de enero y el 31 de diciembre de 1.988 , un estudio analítico de casos y controles con el propósito de identificar factores de riesgo de infección postcesárea. Para el efecto, se seleccionaron como casos 50 pacientes infectadas después de cesárea. Como controles se tomaron 573 pacientes que no presentaron infección postcesárea.

Se observó asociación estadística entre los casos y las variables "Meconio" ["odd ratio":1.9 (p= 0.04)] y "Antibióticos Profilácticos" [odd ratio":0.29. $(\mathbf{p}=\mathbf{0 . 0 0 2})]$.

La presencia de Meconio es factor de riesgo de infección postcesárea. El uso de antibióticos es factor protectivo contra la infección.

Palabras Clave: Riesgo, Infección, Cesárea, Puerperio, Meconio, Antibióticos profilácticos.

SUMMARY: An analitic study of cases an controls to identify risk factors of postcesarean infection was undertaken between January and December 31, 1.988 at Caldas University Hospital, Manizales, Colombia. Fifty patients with postcesarean infections were cases and 573 were controls.

Statistic Association between cases and "Meconio" ["odd ratio":1.9 $(p=0.04)$ ] and "profilactic Antibiotics" ["old ratio": 0.29 (p= 0.002$)]$ variables was found.

"Meconio" is a risk factor in postcesarean section. Profilactic antibiotics use is a protective factor against infection.

KEY WORDS: Risk, Infection, Cesarea, Meconio, Profilactic antibiotics.
\end{abstract}

\section{Introducción}

La cesárea es un procedimiento obstétrico que se ha incrementado en su frecuencia durante los últimos años (1). Este incremento ha suscitado un gran interés y han surgido opiniones discordantes, por tener este procedimiento una mayor morbilidad y mortalidad materna en comparación con el parto por vía vaginal (2-6).

El incremento en el número de cesáreas ha conducido al aumento del índice de infección puerperal y a que se vean cuadros más severos de esta patología (7).

La Endometritis y la Infección de herida quirúrgica, han sido las entidades más frecuentemente observadas (5.8.9) complicando la cesárea. Mediante el presente trabajo se pretendió identificar factores de riesgo de la infección postcesárea en el Hospital Universitario de Caldas, Manizales, Colombia.

\section{Materiales y Métodos}

Se adelantó un estudio analítico de "Casos y Controles" en el Hospital Universitario de Caldas, Manizales, Colombia, en el período comprendido entre el 1 de enero y el 31 de diciembre de 1.988 .

Se consideró "Caso" aquella paciente que presentó uno o más de los siguientes diagnósticos, durante el postoperatorio inmediato: Endometritis, Infección de herida quirúrgica,

* Médico Gineco-Obstetra, Hospital San José, Sogamoso.

** Médico Epidemiólogo, Hospital Universitario de Caldas

*** Profesor Asociado, Departamento de Obstetricia y Ginecología, Universidad de Caldas.
Parametritis, Salpingitis, Peritonitis, Tromboflebitis pélvica, Abscesos pélvicos, Choque séptico. Se excluyeron del estudio pacientes con evidencia de infección obstétrica intraparto.

Como "Control", se incluyeron pacientes que habiendo sido sometidas a cesárea, no sufrieron infección.

Para la recolección de los datos de interés, se utilizó un formulario precodificado, que incluía variables "de persona" y de los "servicios de salud". Fueron exploradas, en total, 18 variables. El formulario en mención fue diligenciado con base en anamnesis y revisión de la historia clínica de la paciente. La tabulación y análisis de los datos se hizo en foma computarizada.

Se planteó como hipótesis nula la no asociación entre las variables independientes exploradas y la variable dependiente "Infección". Esta fue aprobada a un nivel de significancia del 5\% a dos coplas de curva normal.

El plan de análisis incluyó: 1) análisis "crudo" y 2) análisis de regresión logística.

En el análisis "crudo" se cruzó cada una de las variables exploradas con la dependiente "Infección" estimando el "odds ratio" con su respectivo intervalo de confianza a $95 \%$.

\section{Resultados}

En el período comprendido entre enero 1 y diciembre 31 de 1988 se atendieron 4.059 partos en el Hospital Universitario de Caldas. De estos, 736 (18.1\%) por cesárea.

De acuerdo con los criterios de inclusión señalados, se estudiaron 723 pacientes a quienes se realizó cesárea en el período de tiempo mencionado. Se excluyeron 13 pacientes por presentar infección intraparto. 
Fueron identificadas como "Caso" 50 pacientes, (Tabla 1). Como "Control" 673 pacientes.

La patología infecciosa más frecuentemente observada fue la "Endometritis" en 16 pacientes (32\%), seguida de la "Infección de la herida quirúrgica" en 13 pacientes $(26 \%)$. La categoría "Mixta" incluyó más de una patología, (Tabla 1).

Tabla 1.

DISTRIBUCION DE LAS PACIENTES SEGUN TIPO DE INFECCION

\begin{tabular}{|llc|}
\hline Infección & $\mathbf{N}$ & $\%$ \\
\hline Endometritis & 16 & 32.0 \\
İnf. de herida quirúrgica & 13 & 26.0 \\
Mixta & 21 & 42.0 \\
Total & 50 & 100.0 \\
\hline
\end{tabular}

De las 18 variables exploradas mediante análisis "crudo", las variables "Meconio" y "Antibióticos profilácticos" se observaron asociadas a los "Casos" a un nivel alfa del 5\% (Tabla 2).

El "odd ratio" de $1.9(\mathrm{p}=0.04)$ para "Meconio", es indicativo de débil asociación de esta variable con la "Infección", (Tabla 2).

En 7 pacientes "Caso" y en 242 pacientes "Control" se administraron antibióticos profilácticos. (Tabla 2). El "Odd ratio crudo" de 0.29 ( $p=0.002)$ estaría indicando efecto protectivo de los antibióticos contra la "Infección".

Tabla 2 .

\section{CARACTERISTICAS DE CASOS Y CONTROLES}

\begin{tabular}{|c|c|c|c|c|c|}
\hline & Casos & Contr. & O.R. & Rango & P. \\
\hline Edad Media & 23.9 & 25.5 & & & 0.05 \\
\hline Desv. E & 6.4 & 6.3 & & & \\
\hline Mínima & 15.0 & 14.0 & & & \\
\hline Máxima & 40.0 & 44.0 & & & \\
\hline \multicolumn{6}{|c|}{ Controles prenatales } \\
\hline $0-3$ & 21 & 208 & 1.60 & $0.90-2.86$ & 0.15 \\
\hline 4-8 & 29 & 460 & & & \\
\hline \multicolumn{6}{|c|}{ Patología en el embarazo } \\
\hline si & 11 & 195 & & & \\
\hline No & 39 & 478 & 0.69 & $0.35-1.43$ & 0.37 \\
\hline \multicolumn{6}{|c|}{ Edad gestacional (Semanas) } \\
\hline Media & 39.3 & 38.9 & & & 0.05 \\
\hline Mínima & 28 & 24 & & & \\
\hline Máxima & 43 & 45 & & & \\
\hline \multicolumn{6}{|c|}{ Duración trabajo de parto (horas) } \\
\hline$=12$ & 27 & 392 & & & \\
\hline 12 & 23 & 281 & 0.84 & $0.47-1.50$ & 0.05 \\
\hline \multicolumn{6}{|c|}{ Tiempo de ruptura prematura de membranas (horas) } \\
\hline 0 & 17 & 318 & & & \\
\hline $1-6$ & 22 & 192 & & & \\
\hline $7-12$ & 5 & 71 & & & \\
\hline $13-24$ & 3 & 45 & & & \\
\hline 25 YMAS & 3 & 46 & & & 0.23 \\
\hline
\end{tabular}

Número de tactos vaginales

$\begin{array}{lll}0-3 & 41 & 528 \\ 4-6 & 9 & 136 \\ 7 \text { y más } & 0 & 8\end{array}$

Cirujano

$\begin{array}{lll}\text { Infierno } & 9 & 94 \\ \text { Profesor } & 1 & 39 \\ \text { R-1 }(*) & 21 & 295\end{array}$

R-1 (*) $21 \quad 295$

R-2, R-3 19

0.61

Tiempo quirúrgico (horas)

$\begin{array}{llllll}0 & 41 & 466 & & & \\ 21 & 8 & 204 & 2.24 & 1.01-4.78 & 0.05\end{array}$

Tipo de Laparatomía

Mediana 45

Pfannestiel 5

629

$\begin{array}{llll}44 & 0.63 & 0.20-1.65 & 0.24\end{array}$

Tipo de Histerotomía

$\begin{array}{lll}\text { Corporal } & 0 & 1 \\ \text { Seg.-Corporal } & 1 & 13\end{array}$

Seg.-Corporal

Segmentaria $49 \quad 659$

0.96

Número de cesáreas previas

$\begin{array}{rrr}0 & 45 & 497 \\ 1 & 4 & 125 \\ 2 & 1 & 49 \\ 3 & 0 & 2\end{array}$

0.09

Días hospitalización

$\begin{array}{lll}0 & 40 & 506 \\ 1-3 & 9 & 109 \\ 4-7 & 0 & 39 \\ \text { 8-Mas } & 1 & 19\end{array}$

0.36

Hemoglobina (gr./100 ml.)

$\begin{array}{lllllll}10 \text { y más } & 29 & 410 & & & \\ 10 & 4 & 55 & 0.97 & 0.33-2.87 & 1.00\end{array}$

Líquido meconiado

\begin{tabular}{llllll}
$\mathrm{Si}$ & 21 & 185 & & & \\
No & 29 & 486 & 1.90 & $1.02-3.35$ & 0.04 \\
& & & & & \\
bióticos & profilácticos & & & & \\
$\mathrm{Si}$ & 7 & 242 & & & \\
No & 43 & 430 & 0.29 & $0.12-0.68$ & 0.002 \\
\hline
\end{tabular}

(*) R-1 = Residentes de primer año

$\mathrm{R}-2, \mathrm{R}-3$ = Residentes de segundo y tercer año

Un modelo de regresión logística que incluyó un término de interacción entre las variables "Meconio", "Antibióticos" permitió observar, de un lado, independencia entre las dos variables en mención y, de otro, persistencia de la asociación con la variable dependiente "Infección", (Tabla 3).

Tabla 3.

\section{ANALISIS DE REGRESION LOGISTICA}

\begin{tabular}{|c|c|c|c|}
\hline Parámetro & Coeficiente & Error Estándar & $\mathbf{T}$ \\
\hline Constante & -2.54 & 0.20 & -12.22 \\
\hline Meconio & 0.70 & 0.32 & 2.14 \\
\hline Antibióticos & -1.79 & 0.54 & -2.18 \\
\hline Meconio * Antibiótico & -0.14 & 0.84 & -0.17 \\
\hline
\end{tabular}




\section{Discusión}

Se presentó infección puerperal en el $6.9 \%$ de las pacientes estudiadas, lo cual representa un ascenso en el índice de infección en el Hospital Universitario de Caldas en relación con los datos presentados en 1.984 por Durango (10).

La incidencia de infección postcesárea es señalada cor cifras variables de acuerdo con los diferentes estudios ( 11 14), (Tabla 4).

Tabla 4.

INCIDENCIA DE INFECCION POSTCESAREA

\begin{tabular}{|lll|}
\hline Hospital & Año & $\%$ \\
\hline Universitario del Valle (Cali-Colombia) & 1.984 & 3.8 \\
Universitario de Lund (Suecia) & $1.984-1.980$ & 20.0 \\
Central de Boras (Suecia) & $1.978-1.980$ & 13.3 \\
Saint Mary's Health Center (USA) & $1.977-1.978$ & 19.7 \\
\hline
\end{tabular}

Los resultados descritos para "Meconio" están en correspondencia con los hallazgos de otros estudios que han de- mostrado que si bien el meconio es estéril, su constitución puede hacerlo un excelente medio de cultivo (15).

Igualmente, el coeficiente de regresión negativo (-1.26) y significante $(\mathrm{t}=-2.19)$ para "Antibióticos" hallados en el modelo de regresión (Tabla 3 ) está en consonancia con otros estudios $(9,16)$ que confirman la efectividad de la administración profiláctica de antibióticos en la reducción de la infección puerperal postcesárea.

No obstante, Gibbs (8) afirma que los antibióticos no son la panacea y que se hace necesario buscar otras medidas que tiendan a disminuir la tasa de infecciones.

\section{Conclusiones}

La "Endometritis" constituyó la principal patología infecciosa postcesárea en el Hospital Universitario de Caldas.

La presencia de "Meconio" se halló asociada a la infección postcesárea.

La administración de antibióticos profilácticos fue identificada como factor protectivo contra la infección, a niveles altos de significancia estadística.

\section{BIBLIOGRAFIA}

1. Quilligan E. Cesarean section. Clinical Obstetrics and Gynecologic, 1.985. 28 (4) 689

2. Gibs RS, Jones PM, Wilder CJY. The effect of internal monitoring on maternal infection following cesarean section: A prospective study. Obstetrics an Gynecology, 1.978. 25 (2): 193-197.

3. Jones $\mathrm{OH}$. Cesarean section in present-day obstetrics. Presidential address. American Journal of Obstetrics and Gynecologic, 1.976. 126 (5): 521-530.

4. Petitti DB. Maternal mortality and morbidity in cesarean section. Clinical Obstetrics and Gynecology, 1.985. 28 (4): 763-769.

5. Rehu M, Nilsson CG. Risk factors for febrile morbidity associated with cesarean section. Obstetrics and Gynecology, 1.980. 56 (3): 269-273.

6. Rodríguez V. Cesárea en el Hospital San Ignacio. Revista Colombiana de Obstetricia y Ginecología, 1.987. 38: 31-34.

7. Gibbs RS. Clinical risk factors for puerperal infection. Obstetrics and Gynecology. 1.980. 55 (5-9): 178-184.

8. Gibbs RS, St Clair RJ, Castillo MS, Castañeda YS. Bacteriologic effects of antibiotic prophylaxis in hion risk cesarean section. Obstetrics and Gynecolgy. 1.981. 57 (3): 277-282.

9. Rayburn WF, Prophylactic antibiotics during cesarean section: An overview of prior clinical investigations. Clinics in Perinatology. 1.983. 10: 461 .
10. Durango CA, Cesárea en el Hospital Universitario de Caldas durante el período 1.980-1.982. Universidad de Caldas, Colombia, 1.984. Trabajo presentado para optar el título de especialista en Ginecología y Obstetricia.

11. Franco JL, Escobar GS, Hernández H, Medina LR, Paz X. Estudio de casos y controles de los factores de riesgo en la infección postcesárea. Cali, 1.984. Colombia Médica. 1.987. 18 (3): 130-133.

12. Hagglund L, Christensen KK, Christiansen P, Kamme C. Risk factors in cesarean section infection. Obstetrics and Gynecology. 1.983. 62 (2): 145-150.

13. Nielsen TF, Hokegard KH. Postoperative cesarean section morbidity: A prospective study. American Journal of Obstetrics and Gynecolgy. 1.983. $146(8): 911$.

14. Ott WJ. Primary cesarean section: A critical analysis. Obstetrics and Gynecolgy. 1.981. 58 (6): 691-695.

15. Main EK. Meconiun aspiration syndrome. In: Arias F. Highrisk Pregnancy and Deliverity. St. Louis Missoury, the mosby company. $1.984,167$.

16. Galask RP. Changing concepts in obstetrics antibiotic prophylaxis. American Journal of Obstetrics and Gynecology. 1.987. 157 (8): 491-497. 